\title{
AVALIAÇÃO DE USABILIDADE EM INTERFACES WEB: PROCESSOS PARA CRIAÇÃO DE HEURÍSTICAS ESPECÍFICAS
}

\author{
EVALUATION OF USABILITY IN WEB INTERFACES: \\ PROCESSES TO CREATE SPECIFIC HEURISTICS
}

\author{
BERTAGNOLLI, Bruno; Mestrando PPGDesign UDESC; Universidade do Estado de Santa Catarina \\ cb.bruno@gmail.com \\ MAGER, Gabriela; Prof.a. Dr.a. PPGDesign UDESC; Universidade do Estado de Santa Catarina \\ gabrielamager@gmail.com \\ SANTOS; Flávio Anthero Nunes Vianna; Prof. Dr. PPGDesign UDESC; Universidade do Estado de \\ Santa Catarina \\ flavioanvs@hotmail.com
}

\section{Resumo}

O estudo analisa os processos utilizados e resultados obtidos na criação de heurísticas de domínio específico dentro da área de avaliação de usabilidade de interfaces. Para esse fim realizou-se uma revisão sistemática de literatura englobando estudos que abordam a definição de heurísticas como um dos objetivos práticos. Como resultado foi possível traçar processos utilizados na obtenção de heurísticas de domínios específicos assim como os resultados obtidos através das mesmas, o que pode ser utilizado para refinar processos de avaliação de usabilidade através de heurísticas. Conclui-se que os estudos não estruturam claramente o processo para obtenção de heurísticas específicas, o que em domínios complexos pode dificultar processos de avaliação de usabilidade e diminuir a validade dos resultados.

Palavras Chave: Métodos, Heurísticas, Interfaces web

\begin{abstract}
The study analyzes the used processes and results obtained in the creation of specific domain heuristics within the area of evaluation of interface usability. For this purpose a literature review was conducted in studies that addressed the search for heuristics as one of the practical objectives. As a result it was possible to trace processes used to obtain specific domain heuristics as well as the results obtained, which can be used to refine usability evaluation processes through heuristics. It is concluded that the studies do not clearly structure the process to obtain specific heuristics, which in complex domains may hinder usability evaluation processes and decrease the validity of the results.
\end{abstract}

Keywords: Methods, Heuristics, Web interface 


\section{Introdução}

Avaliar interfaces em qualquer fase do projeto - inclusive após concluídas - justifica-se por fornecer aspectos relevantes para a determinação da qualidade de produtos digitais. Fundamentase esta afirmação pelo fato de que um dos fatores mais importantes para se medir a qualidade é o ponto de vista do usuário.

As avaliações de interfaces têm foco na usabilidade, que como afirma Tullis (2008) é considerada a capacidade de o usuário usar um artefato para realizar uma tarefa com sucesso. Para o autor, a visão de medir a usabilidade, deve ser vista como "olhar para toda a experiência do usuário". Dentre as técnicas de avaliação de usabilidade, a avaliação heurística se destaca com algumas vantagens por não envolver usuários, mas sim especialistas, agilizando e simplificando o processo de avaliação.

Nielsen (1994), com um conjunto de 10 heurísticas de usabilidade, é considerado o precursor da técnica, criada com foco no paradigma de usuário único, estacionário, WIMP (Window, Icon, Menu, Pointing device). À época, o uso de tais heurísticas era adequado para a maioria das aplicações, mas o desenvolvimento das tecnologias trouxe desafios na avaliação de interfaces. Assim, pesquisadores começaram a buscar novas soluções para a análise da usabilidade de tais domínios (TSUI et al., 2009).

Considerando a literatura relacionada à avaliação de usabilidade e especificamente ligada à avaliação heurística, este trabalho procura analisar a partir de um processo sistemático de revisão de literatura os processos utilizados e resultados obtidos a partir da criação de heurísticas específicas (HEs) para avaliação de interfaces em sistema web. A escolha sobre o estudo de HEs focadas apenas em interfaces na plataforma computador (e não mobile) procura também demonstrar se as Heurísticas genéricas como as de Nielsen - que foram criadas específicas para atender esse fim - ainda são relevantes. Este trabalho pretende analisar unicamente os processos utilizados e os resultados gerais obtidos na criação de tais heurísticas, não sendo o foco analisar de forma qualitativa e específica as HEs obtidas.

O resultado de uma análise nesse sentido demonstra possíveis caminhos em processos de avaliação de usabilidade que utilizam avaliação heurística, possibilitando uma melhor tomada de decisão por parte do pesquisador.

\section{Métodos de avaliação de usabilidade em interface}

Cybis (2003) conceitua que métodos de avaliação são procedimentos para coletar informações com objetivo de averiguar a interação de usuários com sistemas e visam principalmente avaliar o quesito usabilidade. Os testes de usabilidade possuem duas abordagens, uma envolvendo testes formais realizados como verdadeiras experiências (a fim de confirmar ou refutar hipóteses específicas), e outra que emprega um ciclo iterativo de testes (destinados a expor deficiências de usabilidade ou moldar o produto em questão).

Souza, Leite, Prates \& Barbosa (1999) colocam que a escolha do método que deve ser aplicado em um caso específico depende de vários fatores, como o que se deseja avaliar, disponibilidade de pessoas especialistas, ambiente e equipamento para aplicação do teste, acesso aos usuários entre outros.

Tratando-se da classificação de métodos de avaliação de usabilidade, de forma geral pode- 
se dividi-los em dois grupos (CYBIS, 1997; JORDAN, 1998):

A. Métodos que envolvem usuários:

Métodos Empíricos/objetivos: buscam constatar problemas partindo da observação do usuário interagindo com o sistema;

Métodos Prospectivos: envolvem o usuário buscando sua opinião sobre a interação com o sistema. São baseados na aplicação de questionários/entrevistas com o usuário para avaliar sua satisfação ou insatisfação em relação ao sistema e sua operação.

B. Métodos que não envolvem usuários:

Métodos preditivos / diagnósticos / analíticos: envolvem apenas especialistas. Buscam prever erros de projeto de interfaces, sendo realizados geralmente pelos projetistas ou por especialistas em usabilidade com experiência em avaliação.

Dentro da categoria de métodos preditivos, onde se encaixa o objeto de estudo desse trabalho, as ferramentas de avaliação variam no sentido de como os julgamentos são efetuados e em quais critérios espera-se que o avaliador baseie seus julgamentos.

Cybis (1998), Wharton (1994) e Jordan (1998) colocam que entre as ferramentas preditivas estão os Checklists (Listas de Verificação), Percurso Cognitivo, Inspeção de Conformidade, Análise Hierárquica de Tarefas, Avaliação de Peritos e a Avaliação Heurística.

\section{Avaliação heurística e aplicação}

Segundo Preece et. al. (2007), o termo heurística "[...] enfatiza que algo deve ser feito com esses princípios, quando aplicados a um dado problema. Em particular, precisam ser interpretados no contexto do design, utilizando-se de experiências já realizadas [...]".

Utilizando-se de critérios heurísticos, a avaliação heurística é considerada um dos métodos preditivos (sem usuários) mais populares, por ser considerada barata, rápida e obter resultados relevantes (BONIFÁCIO et. al., 2010; PRATES E BARBOSA, 2003).

Assim, o conceito de avaliação heurística é inspecionar sistematicamente a interface do usuário para examinar se o design está de acordo com regras de usabilidade conhecidas (WHITEHEAD, 2006; PRATES et. al., 2014). Como objetivo avaliativo, busca-se essencialmente a avaliação de aspectos relacionados com a usabilidade de interfaces a fim de detectar problemas de projeto e fazer recomendações para a eliminação de tais problemas.

Diversas linhas e orientações desses critérios podem ser encontradas na literatura, como os de Dias (2007), Cybis (2007), Jordan (2001); Pressman (2004), Shneiderman (2005), os Oito critérios de Bastien \& Scapin (1993) e as dez heurísticas de Nielsen (1994, 2003, 2007). Todos esses estudos são baseados em experiências empíricas dos pesquisadores, derivadas da experiência profissional, teoria e senso comum, existindo diversos pontos de convergência, sendo muitas vezes complementares. Shneiderman (2005) coloca que tais princípios visam o aumento de produtividade por indicar ao final de um processo de avaliação apontamentos sobre melhorias nas interfaces.

Embora tenham sido utilizados para avaliação de interfaces diversas, autores como Nielsen (1994) e Shneiderman (2005) enfatizam que tais princípios apresentados devem ser interpretados, refinados e estendidos para cada ambiente, de acordo com a plataforma, domínio de aplicação e necessidade específica. 


\subsection{Aplicação}

A avaliação heurística é realizada por meio de um conjunto pequeno de especialistas que, separadamente, avaliam a interface confrontando-a com as regras heurísticas escolhidas. Objetivase identificar eventuais erros que comprometam a usabilidade, em sessões que duram cerca de uma ou duas horas. Os resultados podem ser documentados em relatórios individuais por cada avaliador, ou em um grande relatório feito por um observador a partir da observação dos avaliadores e seus relatos.

Recomenda-se que a interface seja analisada pelos critérios escolhidos pelo menos duas vezes. A primeira análise deve realizar os principais fluxos de tarefas, verificando a interação no âmbito geral do sistema. A segunda análise deve ser concentrada em elementos de interface específicos, compreendendo como eles se encaixam na interface como um todo.

Cada problema deve ser explicado em detalhes, usando como referência os princípios de usabilidade estabelecidos na lista de heurísticas. Para que tais problemas possam ser relacionados ao impacto que causam na interface, Prates et. al. (2014) coloca que cada avaliador deve definir a localização e o grau de severidade de cada problema encontrado, o que ao final aponta para qual tipo de intervenção deve ser tomada.

\section{Materiais e métodos}

Baranauskas et. al. (2003) destaca que podem ser formuladas heurísticas voltadas para a avaliação de interfaces de determinada categoria de produtos por meio de heurísticas derivadas da avaliação de produtos similares. Tais heurísticas podem ser consideradas um suplemento para as heurísticas gerais - HGs. De forma geral, a criação de heurísticas é feita através da avaliação de sistemas existentes, e pelo desenvolvimento de princípios que descrevem os problemas de usabilidade que são encontrados por especialistas.

Consubstanciando tal posicionamento, a literatura traz diversos trabalhos onde o processo de geração e adaptação de heurísticas é desenvolvido como um método para aprimorar a etapa de avaliação. Tais estudos buscam obter as chamadas "heurísticas específicas - HEs", ou pelo menos referendar o melhor conjunto de Heurísticas para a avaliação dos sistemas em questão.

Embora seja reconhecida essa necessidade, a forma ou o processo para definição de tais heurísticas por vezes não é claro, o que motivou uma pesquisa buscando analisar processos utilizados na criação de HEs assim como os resultados obtidos.

Assim, partiu-se para uma revisão de literatura sistemática que englobou trabalhos com foco principal no desenvolvimento ou no processo de refinamento de heurísticas para domínios específicos. Tal busca ocorreu nas bases de dados Scopus, Science Direct, Proquest e Sucupira. Para uma primeira busca, termos como "interface"; "avaliação de usabilidade"; "conjunto heurístico" e "checklist heurístico" foram utilizados, assim como os equivalentes em inglês.

O processo de busca, seleção e análise de trabalhos ocorreram no mês de outubro de 2017. Realizou-se um detalhamento para os campos de busca, de acordo com a sintaxe que permitisse que cada um dos repositórios apresentasse os melhores resultados de acordo com os filtros, trazendo um equilíbrio na relação quantitativa $x$ relação com o escopo de busca. 0 detalhamento é apresentado na Tabela 1. 
TABELA 1: Campos de Busca da Revisão Sistemática da Literatura

\begin{tabular}{|c|c|}
\hline Base de dados & Strings de busca \\
\hline Scopus & $\begin{array}{l}\text { ("web interface") AND ("usability heuristics" OR "usability heuristic") AND } \\
\text { (methodology OR design OR "formal process" OR process) AND ("heuristic } \\
\text { evaluation") }\end{array}$ \\
\hline Proquest & ("interface") AND ("usability heuristics" OR "usability heuristic") \\
\hline Sucupira & ("interface") AND ("usability heuristics" OR "usability heuristic") \\
\hline Google Scholar & $\begin{array}{l}\text { ("web interface") AND ("usability heuristics" OR "usability heuristic") AND } \\
\text { (methodology OR design OR "formal process" OR process) AND ("heuristic } \\
\text { evaluation") }\end{array}$ \\
\hline Science Direct & (“heuristic evaluation") AND (website) \\
\hline
\end{tabular}

Fonte: Autor

Como critérios de inclusão e exclusão, considerou-se para a revisão da literatura artigos que estivessem dentro dos seguintes critérios:

TABELA 2: Critérios de inclusão e exclusão adotados para a revisão sistemática

\begin{tabular}{|l|l|}
\hline \multicolumn{1}{|c|}{ Critérios de inclusão } & \multicolumn{1}{c|}{ Critérios de exclusão } \\
\hline $\begin{array}{l}\text { - Estudos onde a formulação de heurísticas específicas } \\
\text { era uma das etapas do trabalho; }\end{array}$ & $\begin{array}{c}\text { - Estudos que apenas aplicaram modelos heurísticos } \\
\text { prontos; }\end{array}$ \\
$\begin{array}{l}\text { - Estudos que trouxessem no título ou resumo a } \\
\text { proposta de avaliação heurística para avaliação de }\end{array}$ & $\begin{array}{c}\text { - Estudos que não deixavam claro o processo para } \\
\text { escolha das heurísticas; }\end{array}$ \\
websites; & $\begin{array}{l}\text { - Estudos voltados para interfaces mobile ou aplicações } \\
\text { não site/software; }\end{array}$ \\
- Estudos dos últimos 10 anos; & $\begin{array}{l}\text { Estudos que voltados exclusivamente a criar/validar } \\
\text { - Estudos diversos (teses, artigos, periódicos, etc.); }\end{array}$ \\
\hline
\end{tabular}

Fonte: Autor

Inicialmente encontraram-se como resultado das buscas 566 trabalhos, como mostra a Tabela 2. Numa fase inicial foram revisados os títulos, abstracts e palavras chaves em relação aos critérios de inclusão. Estudos irrelevantes em relação a esses critérios ou relevantes em relação aos critérios de exclusão não foram lidos. A maioria dos estudos não trazia claramente o processo de desenvolvimento ou refinamento das heurísticas utilizadas, e, portanto, foram descartados. Isso resultou na análise de 40 trabalhos, que foram revisados mais a fundo, observando além do título, do abstract e das palavras chaves o texto principal, novamente aplicando os critérios de inclusão e exclusão. 
TABELA 2: Base de dados e resultado da busca.

\begin{tabular}{|l|l|l|}
\hline \multicolumn{1}{|c|}{ Base de dados } & \multicolumn{1}{c|}{ Número de artigos encontrados } & \multicolumn{1}{c|}{ Primeira Seleção } \\
\hline Scopus & 69 & 9 \\
\hline Proquest & 169 & 17 \\
\hline Sucupira & 207 & 6 \\
\hline Google Scholar & 216 & 25 \\
\hline Science Direct & 121 & 8 \\
\hline
\end{tabular}

Fonte: Autor

Ao final, 16 estudos foram considerados com informações suficientes e relevantes para uma leitura e análise aprofundada, sendo tabulados conforme a tabela 3. A análise estruturada focou em responder as questões do estudo, como a classificação e descrição dos métodos utilizados e resultados obtidos no esforço para criação de tais heurísticas.

TABELA 3: Estudos escolhidos após a seleção.

\begin{tabular}{|c|c|c|}
\hline Cód. & Autor do estudo / Ano & Fonte \\
\hline$(1)$ & MUÑOZ et al. (2012) & \multirow{5}{*}{ Scopus } \\
\hline$(2)$ & PAZ et al. (2014) & \\
\hline$(3)$ & ALSUMAIT et. al (2010) & \\
\hline$(4)$ & LEE at. Al. (2016) & \\
\hline$(5)$ & DIAZA et al. (2017) & \\
\hline (6) & PRIBEANU (2014) & \multirow{2}{*}{ PROQUEST } \\
\hline (7) & HAIRIRI et. al. (2011) & \\
\hline (8) & CARRARE (2014) & Sucupira \\
\hline (9) & ALMEIDA et. al. (2013) & \multirow{6}{*}{$\begin{array}{l}\text { Google } \\
\text { Scholar }\end{array}$} \\
\hline (10) & PADARATZ (2014) & \\
\hline$(11)$ & ALVEZ (2014) & \\
\hline$(12)$ & AMSTEL (2011) & \\
\hline (13) & GONZALES (2007) & \\
\hline (14) & SANTINHO (2001) & \\
\hline (15) & ALLEN et. al. (2005) & \multirow{2}{*}{$\begin{array}{l}\text { Science } \\
\text { Direct }\end{array}$} \\
\hline$(16)$ & GUMUSSOY (2016) & \\
\hline
\end{tabular}

FONTE: Autor

A partir do conjunto selecionado, foi possível mapear, organizar e traçar pontos chaves em comum que permitiram traçar um panorama dos processos utilizados.

\section{Resultados}

Para responder ao problema de estudo, formularam-se questionamentos que pudessem responder as duas questões de pesquisa: 
Questão de pesquisa 1: Quais são os processos utilizados na literatura para a criação de HEs?

- Como tais processos podem ser classificados e descritos?

- Tais processos utilizam algum modelo ou base heurística? Quais seriam esses modelos?

- Quais os domínios de aplicação das HEs?

- As HEs criadas passaram por validação?

- Qual o processo utilizado para validação?

Questão de pesquisa 2: Quais os resultados obtidos a partir da criação de HEs?

- Qual o resultado prático do estudo?

\subsection{Abordagem sobre processos utilizados na construção de heurísticas}

Para esse tópico, realizou-se a análise das abordagens utilizadas nos estudos, buscando a concepção de HEs. Considerando a diversidade encontrada, duas classificações foram adotadas:

TABELA 4: Métodos formais e informais identificados na análise dos estudos

\begin{tabular}{|c|c|c|c|c|}
\hline Métodos formais & \multicolumn{4}{|c|}{ Métodos Informais } \\
\hline Baseado em Método & $\begin{array}{l}\text { Revisão de } \\
\text { literatura }\end{array}$ & $\begin{array}{l}\text { Baseados em } \\
\text { problemas de } \\
\text { usabilidade }\end{array}$ & $\begin{array}{l}\text { Baseados em } \\
\text { entrevista }\end{array}$ & Mistos \\
\hline$(1) ;(2) ;(5)$ & $\begin{array}{c}(3),(6),(7),(8),(10), \\
(11)\end{array}$ & $\begin{array}{c}(12),(13),(15), \\
(16)\end{array}$ & (4), (9) & Mistos (14) \\
\hline $\begin{array}{l}\text { 1- Etapa exploratória: explora } \\
\text { as aplicações específicas que } \\
\text { exigem novas heurísticas. } \\
2 \text { - Etapa descritiva: } \\
\text { reexamina o significado da } \\
\text { usabilidade e suas } \\
\text { características. } \\
3 \text { - Etapa de correlações: } \\
\text { identifica os recursos que as } \\
\text { heurísticas de usabilidade } \\
\text { para aplicações específicas } \\
\text { devem ter. } \\
4 \text { - Etapa de execução: } \\
\text { especifica novas heurísticas. } \\
5 \text { - Etapa de validação: avalia } \\
\text { o conjunto de heurísticas } \\
\text { definido na etapa } 4 . \\
6 \text { - Fase de refinamento: } \\
\text { refina o conjunto de } \\
\text { heurísticas definido na etapa } \\
4 .\end{array}$ & $\begin{array}{l}1 \text { - Revisão de } \\
\text { literatura do objeto } \\
\text { de estudo. } \\
2 \text { - Revisão de } \\
\text { literatura de } \\
\text { modelos heurísticos } \\
\text { existentes (gerais } \\
\text { ou específicos). } \\
3 \text { - Testagem de } \\
\text { usabilidade das } \\
\text { interfaces e } \\
\text { definição das } \\
\text { necessidades (feita } \\
\text { em testes por grupo } \\
\text { de especialistas do } \\
\text { domínio). } \\
4 \text { - Correlações } \\
\text { entre necessidades } \\
\text { e heurísticas. } \\
5 \text { - Conjunto } \\
\text { heurístico refinado. }\end{array}$ & $\begin{array}{l}1 \text { - Com base em } \\
\text { um modelo } \\
\text { heurístico ou o } \\
\text { conhecimento do } \\
\text { próprio avaliador, } \\
\text { realiza-se } \\
\text { avaliação } \\
\text { heurística de } \\
\text { diversas } \\
\text { interfaces do } \\
\text { domínio em } \\
\text { busca de falhas. } \\
2 \text { - Levantamento } \\
\text { de severidade e } \\
\text { categorização de } \\
\text { problemas } \\
\text { encontrados. } \\
3 \text { - Tabulação dos } \\
\text { resultados e } \\
\text { redação das } \\
\text { heurísticas. }\end{array}$ & $\begin{array}{l}1 \text { - Escolha/ } \\
\text { construção de um } \\
\text { modelo heurístico } \\
\text { (construído/expan } \\
\text { dido com base na } \\
\text { literatura). } \\
\text { 2- Condução de } \\
\text { avaliação das } \\
\text { interfaces por } \\
\text { entrevista com } \\
\text { base no modelo } \\
\text { heurístico } \\
\text { escolhido. } \\
3 \text { - Avaliação dos } \\
\text { resultados. } \\
4 \text { - Refinamento } \\
\text { do modelo da } \\
\text { etapa } 1 .\end{array}$ & $\begin{array}{l}1 \text { - Avaliação } \\
\text { Heurística com } \\
\text { base em um } \\
\text { modelo. } \\
2 \text { - Utilização de } \\
\text { outros métodos } \\
\text { de avaliação de } \\
\text { usabilidade } \\
\text { (walktroughs, } \\
\text { construção de } \\
\text { cenários, think } \\
\text { aloud, etc.). } \\
3 \text { - Verificação de } \\
\text { problemas } \\
\text { obtidos em cada } \\
\text { método. } \\
4 \text { - Conformação } \\
\text { dos resultados } \\
\text { obtendo uma lista } \\
\text { de heurísticas. }\end{array}$ \\
\hline
\end{tabular}

Fonte: Autor 
A. Métodos formais: considera a utilização atividades sistemáticas e racionais já referenciados e validados pela literatura (MARCONI, LAKATOS, 2010, p. 65). Como método formal, o de Rusu et. al. (2011) é utilizado em três estudos.

B. Métodos informais: considera-se aqui os estudos que seguem uma abordagem empírica e própria de atividades e etapas, desenvolvidas no próprio estudo sem referências a literatura formal. A análise e o agrupamento de tais processos permitiram uma classificação definida em quatro abordagens principais:

- Baseados na revisão de literatura: utilizam a busca de modelos heurísticos (templates) como base para construção de HEs. Os modelos podem ser genéricos ou ter relação com as áreas do domínio em questão. As heurísticas contidas em tais conjuntos podem passar por um processo de comparação, harmonização, escolha, descarte, ampliação ou refinação, chegando-se ao conjunto de critérios ideal;

- Baseados em problemas de usabilidade: utilizam como ponto de partida a utilização de testes de usabilidade com especialistas ou usuários em busca de identificação de problemas e posterior redação de HEs que possam cobrir tais problemas;

- Baseados em entrevistas: utilizam como ponto de partida principal a condução de entrevistas com usuários finais como forma de realizar avaliação da interface em questão, buscando identificar problemas e identificar novas heurísticas que possam cobrir tais problemas;

- Baseados em métodos mistos: utilizam uma mistura de processos para definição de HEs, de forma a obter um conjunto derivado de várias perspectivas, aumentando a chance de efetividade do conjunto criado.

Tal classificação é apenas uma forma de abordar e clarificar os resultados, pois, tratando-se de processos informais, há uma dificuldade de definir de forma mais apurada as etapas. Por esse motivo, levou-se em consideração a técnica com maior destaque e predominância em cada estudo para realizar a classificação. O gráfico 1 mostra que a maioria dos trabalhos aborda de forma informal a busca por HEs.

GRÁFICO 1: Abordagem metodológica para definição de HEs nos estudos

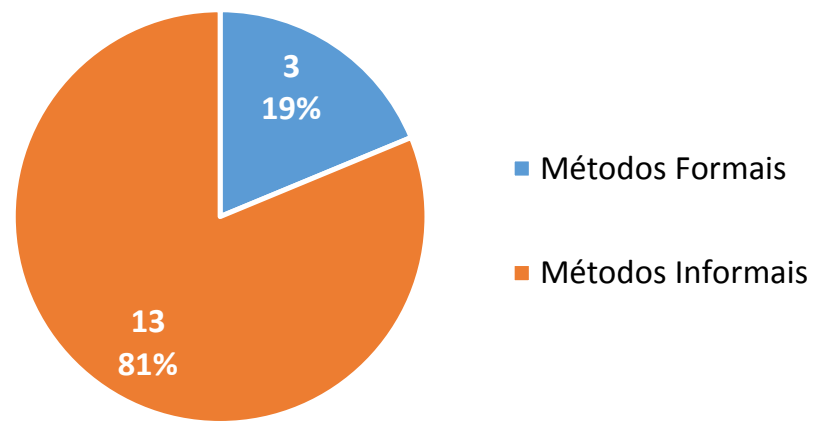

Fonte: Autor

A tabela 4 mostra a classificação dos métodos encontrados e uma visão geral das etapas utilizadas. 
GRÁFICO 2: Porcentagem de trabalhos baseados em cada método

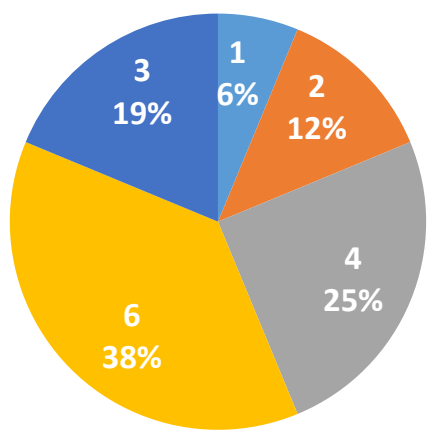

Baseados em Técnicas Mistas

Baseados em entrevistas

Baseados em problemas de usabilidade

Baseados em revisão de literatura

Baseados em métodos (formais)

Fonte: Autor

Outra análise realizada é se tais estudos utilizam alguma base heurística como ponto de partida ou reflexão para o levantamento de HEs. Verificou-se que modelos de HGs consolidados na literatura, embora apontados como não adequados para domínios específicos, ainda tem importância como base teórica, conforme apresentado no gráfico 3. O conjunto de heurísticas de Nielsen (1994) é referenciado por grande parte dos trabalhos, tanto nos estudos que utilizam métodos informais como formais.

GRÁFICO 3: Porcentagem de estudos que partem de um modelo

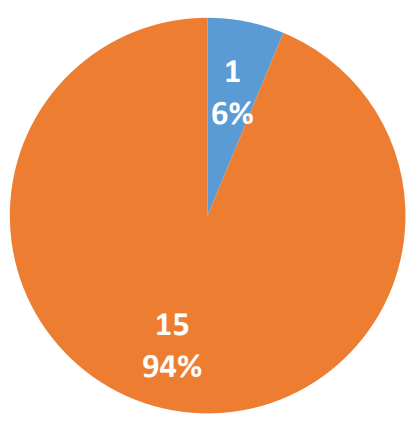

Estudos que não partem de um modelo Heurístico

Estudos que partem de um modelo Heurístico

Fonte: Autor

O gráfico 4 aponta que a utilização de um modelo heurístico é um padrão recorrente nos estudos. A utilização de vários modelos aparece em segundo lugar, influenciado pelo número de estudos que utilizam revisão de literatura, que tem como base a fundamentação teórica em heurísticas para reforçar a consistência do conjunto criado. 
GRÁFICO 4: Quantidade de modelos heurísticos utilizados como ponto de partida dos estudos

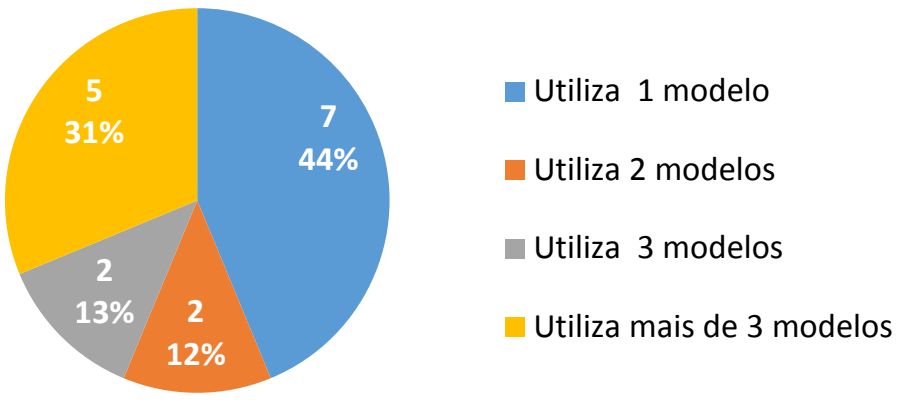

Fonte: Autor

Em relação às referências a modelos heurísticos clássicos, o conjunto das 10 Heurísticas de Nielsen é a mais utilizada, mostrando sua ainda relevância dentro dos estudos de usabilidade. Em segundo lugar aparecem os modelos de domínios específicos, apontando para o maior desenvolvimento de literatura ergonômica focada no desenvolvimento de heurísticas aplicadas.

GRÁFICO 5: Referências a modelos heurísticos utilizados como ponto de partida dos estudos

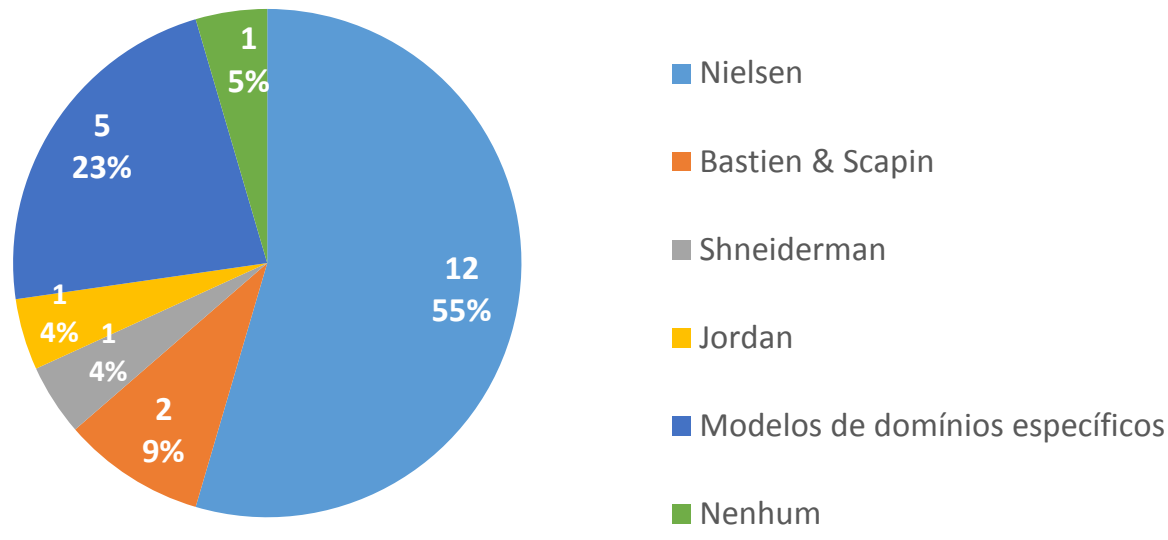

Fonte: Autor

Buscou-se compreender a profundidade do domínio das áreas das interfaces em relação à necessidade de criação de HEs. Os domínios dos sistemas foram classificados em cinco áreas: entretenimento; negócios/comercial; educação; saúde; institucional. Alguns conjuntos de HEs são trabalhados de forma mais genérica para o domínio, principalmente os ligados à educação. Outros apontam para abordagens mais empíricas e experimentais, devido à profundidade, complexidade e especificidade da área, como os domínios ligados a saúde/área médica (Gráfico 6). 
GRÁFICO 6: Áreas de domínio específico dos estudos

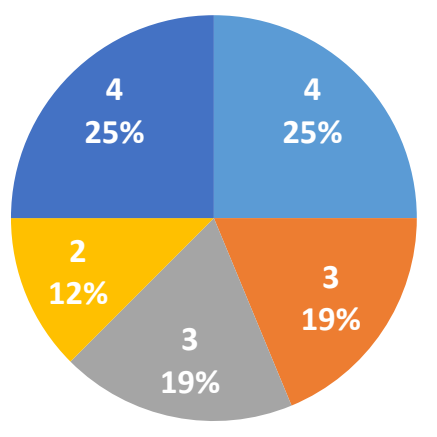

Entretenimento
Comercial / negócios
Educação
Saúde
Institucional

Fonte: Autor

Outra questão analisada foi a validação das HEs. Para essa questão ser afirmativa, os estudos deveriam após a definição das HEs promover testes a fim de averiguar a eficiência e eficácia do conjunto. O objetivo é analisar os resultados quanto à detecção de problemas de usabilidade, indicando a efetividade do conjunto para avaliação de domínios equivalentes (Gráfico 7).

GRÁFICO 7: Estudos e validação das HEs criadas

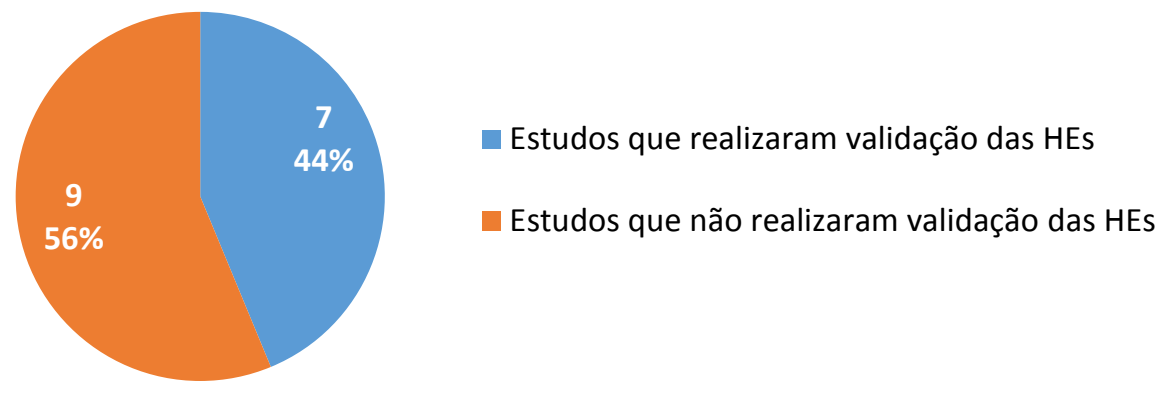

Fonte: Autor

Considerando os estudos que promoveram validação, procurou-se verificar como se realizou tal processo. Na maioria dos casos, verificou-se similaridade nas etapas. De forma geral, podem-se compilar os procedimentos de validação nos seguintes passos:

A. Após a criação das HEs, um grupo formado por pelo menos 3 especialistas (especialistas no domínio específico ou/e em usabilidade) realizam avaliações heurísticas utilizando as HEs propostas e utilizando outro modelo (ex: Nielsen). Interfaces diferentes relativas ao domínio em questão são utilizadas como objetos de avaliação;

B. Confronta-se o número de problemas de usabilidade identificados e os não identificados por cada modelo heurístico;

C. A partir dos resultados da análise obtidos no passo 2, verifica-se a eficiência do novo modelo. Caso sejam identificadas falhas, volta-se a uma etapa de discussão e refinamento entre especialistas.

\subsection{Abordagem sobre resultados na criação de HEs}

Como forma de responder a segunda questão de pesquisa, realizou-se também a análise dos resultados obtidos nos estudos, com relação específica as heurísticas resultantes de todo o 
processo desenvolvido - em uma abordagem qualitativa (classificação) e quantitativa (quantidade de HEs geradas ao final). Avaliou-se que embora alguns estudos tenham como foco a busca de HEs, o resultado final é um conjunto refinado de heurísticas a partir de critérios consolidados na literatura. Isso reflete de certa forma a relevância que conjuntos heurísticos consolidados ainda possuem sobre a questão de avaliação de usabilidade de certos domínios.

Para a classificação qualitativa dos resultados, identificou-se características que permitiram o seguinte agrupamento:

- Heurísticas mantidas: obtidas diretamente de conjuntos utilizados como referência nos estudos, sem alterações. Respondem às questões de nível genérico em relação à usabilidade da interface;

- Heurísticas adaptadas/derivadas: criadas a partir da alteração de alguma heurística já existente e listada na literatura. Respondem às questões de nível intermediário em relação à usabilidade da interface;

- Heurísticas novas: não são baseadas em nenhum modelo anterior, sendo criadas de acordo com a necessidade levantada no processo. Respondem às questões de nível específico em relação à usabilidade da interface em questão, podendo ser consideradas as verdadeiras HEs.

GRÁFICO 8: Porcentagem de estudos que apresentam heurísticas mantidas, adaptadas e novas

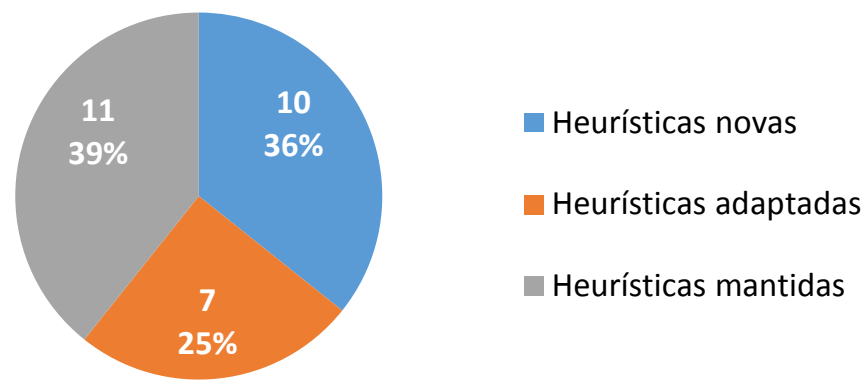

Fonte: Autor

A abordagem quantitativa analisou o volume final de critérios heurísticos gerados pelos estudos. Conforme recomendação de literatura do método heurístico, o número total de heurísticas utilizadas em avaliações de usabilidade deve ser baixo, de forma a facilitar os processos avaliativos. O intervalo quantitativo de HEs identificados foi de $5-25$, sendo que metade dos estudos criou conjuntos no intervalo entre 11 - 18 HEs (Gráfico 9).

GRÁFICO 9: Quantitativo de HEs geradas por percentual de estudos

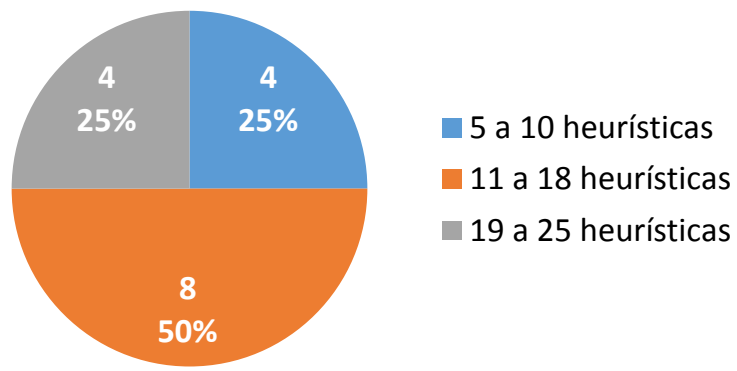

Fonte: Autor 


\section{Conclusão e trabalhos futuros}

O desenvolvimento do presente estudo permitiu uma pesquisa que se aprofundou nos métodos de avaliação de usabilidade preditivos e principalmente no levantamento dos processos utilizados na literatura para obtenção de heurísticas voltadas para avaliação de interfaces de domínios específicos. Tal levantamento possibilitou a obtenção de dados mais consistentes dentro de processos de avaliação de usabilidade.

Os métodos preditivos têm diversas vantagens quando utilizados como ferramenta de avaliação de usabilidade em interfaces - são considerados de fácil utilização, práticos e baratos. A avaliação heurística, como um desses métodos tem sua importância consolidada na literatura. Nielsen, Bastien e Scapin, Schneiderman e Jordan fundamentam $72 \%$ dos artigos pesquisados. Os modelos consolidados são bastante úteis para avaliações. No entanto, deve-se considerar que o contexto de criação de tais modelos, entre fim da década 1990 e início da década de 2000, é de um período onde os desafios em relação à usabilidade de interfaces eram menos complexos face às tecnologias, aos domínios e público aos quais eram dirigidas tais tecnologias. Atualmente, frente às complexidades impostas pela disseminação de interfaces humano-computador para os mais diversos fins, a definição de critérios heurísticos específicos é fundamental para a obtenção de resultados precisos em avaliações de usabilidade. Nos artigos pesquisados, verificou-se categorias de utilização de heurísticas que podem ser agrupadas em três grupos: os que mantiveram heurísticas consolidadas na literatura (39\%), os que adaptaram as heurísticas consolidadas (25\%) e os que desenvolveram novas heurísticas adaptadas às especificidades necessárias (36\%).

Entretanto, mesmo considerando essa necessidade e a importância da criação de heurísticas específicas, verificou-se que dos 16 estudos analisados, em 13 (81\%), o processo para alcançá-las ainda não é estruturado, sendo muitas vezes bastante variado. Em processos de avaliação de usabilidade de interfaces complexas, isso pode trazer desafios na obtenção e validade dos resultados. No entanto, também se observou padrões e passos similares entre os trabalhos, o que permitiu uma estratificação que indicou etapas que podem ser utilizadas para uma melhor organização na busca de heurísticas para domínios específicos. Observou-se que a necessidade de cada projeto deve ser avaliada conforme o contexto e recursos da pesquisa.

Como trabalho futuro, entende-se que um aprofundamento e desdobramento deste trabalho pode possibilitar a criação e a validação de um método que poderá ser utilizado para a definição de heurísticas para qualquer domínio específico, além disso, que possa ser realizado um estudo que trate de uma análise qualitativa das HEs criadas.

\section{Referências}

AMARAL, Daniela. Adequação da Interface do SIA-AE aos Critérios Ergonômicos e de Usabilidade. Joinville, 2006, 153f. Dissertação (Bacharelado em Ciência da Computação) - Universidade do Estado de Santa Catarina.

AMSTEL, F. M. C. Heurísticas para Avaliação de Interfaces de Portais Universitários. 2011. Disponível em:<https://research.utwente.nl/en/publications/heuristicas-para-avaliacao-de- 
interfaces-de-portais-universitario >. Acesso em: 10 out. 2017.

ASSOCIAÇÃO BRASILEIRA DE NORMAS TÉCNICAS. NBR ISO 9241: ergonomia da interação humanosistema. Parte 151: orientações para interfaces de usuários da World Wide Websistemas interativos. Rio de Janeiro: ABNT, 2011.

BASTIEN, C.; SCAPIN, D.. Ergonomic criteria for evaluating the ergonomic quality of interactive systems. Behaviour \& Information Technology. Londres: v.16, n.4/5, p.220-231, 1997. CYBIS, W. A.. Desenvolvimento de técnicas de inspeção ergonômica de sistemas interativos. 1997. Disponível em: <http://www.labiutil.inf.ufsc.br/apostila.htm>. Acessado em: 10 out 2017.

CYBIS, W. de A. Ergonomia de Interfaces Humano-Computador. Florianópolis, 2003.

CYBIS, W. BETIOL, A. H.; FAUST R.. Ergonomia e usabilidade: conhecimentos, métodos e aplicações. São Paulo: Novatec, 2007.

DIAS, Cláudia. Usabilidade na web: criando portais mais acessíveis. 2. ed. Rio de Janeiro: Alta Books, 2007.

GONZALEZ, M.P., GRANOLLERS T., PASCUAL, A., LORÉS J..Testing website usability in Spanishspeaking academia through heuristic evaluation and cognitive walkthroughs. Int J Univers Comp Sci, v. 14, n. 9, p. 1513-1527, 2008.

JORDAN, Patrick W. An Introduction to Usability. Londres: Taylor \& Francis, 2001.

NIELSEN, J. "Heuristic Evaluation”, in Mack, R. \& Nielsen, J. (eds.) Usability Inspection Methods. New York, NY: John Wiley \& Sons, 1994, 25-62.

Publishing. 2000.

Designing Web Usability: The Practice of Simplicity. Indianapolis: New Riders

. Ten Usability Heuristics. 2001. Disponível em:

<http://www.useit.com/papers/heuristic/heuristic_list.html>. Acesso em 15 de Novembro de 2017.

NIELSEN, J.; MOLICH, R.. Heuristic evaluation of user interfaces. 1990. Disponível em: <http://www.acm.org/dl>. Seattle, Washington, United States. Acesso em dez. 2017.

NIELSEN, Jakob. 10 Usability Heuristics for User Interface Design, 1994. Disponível em: < http://www.nngroup.com/articles/ten-usability-heuristics/> . Acesso em: 10 nov. 2017

PARADATZ, Aline. Avaliação Heurística de Usabilidade em Museus Virtuais de Moda. DAPesquisa, v.9, n.12, p 01 - 16, dezembro 2014. Disponível em:

<http://www.revistas.udesc.br/index.php/dapesquisa/article/download/5019/3963.>. Acesso em: 8 outubro de 2017 
PELOGI A. P. S. , GUIMARÃES M. P. , BARSOTTINI C. G. N.. Heurísticas Contextuais para Portais Universitários. II Congresso Brasileiro de Informática na Educação (CBIE). XXIV Simpósio Brasileiro de Informática na Educação (SBIE). 2013. Disponível em:

https://www.researchgate.net/publication/299684255_Heuristicas_Contextuais_para_Portais_Un iversitarios. Acesso em: 15 set. 2017.

PREECE, Jenny; ROGERS, Yvonne; SHARP, Helen; BENYON, David; HOLLAND, Simon \& CAREY, Tom. Human-Computer Interaction. ADDISON-WESLEY, The Open University, 1995.

PRESSMAN, R. S. Software engineering: a practioner's approach. Nova York: McGraw-Hill, Proceedings of the 10th Brazilian Symposium on Human Factors in Computing Systems and the 5th Latin American Conference on Human-Computer Interaction. 2004

ROCHA, H. V.; BARANAUSKAS, M. C. C. Design e avaliação de interfaces humanocomputador. [s.I.] Unicamp, 2003.

RUSU, C., RONCAGLIOLO, S., RUSU, V., COLLAZOS, C., A Methodology to Establish Usability Heuristics. In: Proceedings of The Fourth International Conference on Advances in ComputerHuman Interactions, 2011.

SANTINHO, M. Avaliação Heurística e testes com utilizadores: dois métodos, dois resultados. Disponível em:< https://paginas.fe.up.pt/ ei98016/ES/docs/set2001.pdf>. Acesso em 30 set. 2017.

SHNEIDERMAN, B.; PLAISANT, C. Designing the user interface: strategies for effective humancomputer interaction. 4. ed. Addison Wesley Publishing Company, 2004

SOUZA, C. S. de; LEITE, J. C.; PRATES, R. O.; BARBOSA, S. D. J. Projeto de interfaces de usuário: perspectivas cognitivas e semióticas. Anais da Jornada de Atualização em Informática, XIX Congresso da Sociedade Brasileira de Computação, Rio de Janeiro, 1999.

TSUI K. M., Abu-Zahra K., CASIPE R., M'Sadoques J., DRURY J. L. A process for developing specialized heuristics: Case study in assistive robotics (Technical Report No. 2009-11). Lowell: University of Massachusetts Lowell. 2009.

TULLIS, Tom; ALBERT, Bill. Measuring the user experience: Collecting, Analyzing, and Presenting Usability Metrics. Burlington: Morgan Kaufman, 2008.

WHARTON, C., RIEMAN, J., LEWIS, C. and POLSON, P. The Cognitive Walkthrough Method: A Practitioner's Guide. In Nielsen, J., and Mack, R.L. (Eds.), Usability Inspection Methods, John Wiley \& Sons, New York, NY, 1994.

WHITEHEAD, C.. Evaluating Web Page and Web Site Usability. 2006. Disponível em:<http://delivery.acm.org/10.1145/1190000/1185637/p788- 
whitehead.pdf?key1=1185637\&key2=5262873711\&coll=\&dl=GUIDE\&CFID=15151515\&CFTOKEN= 6184618>. Consultado em 01 de Novembro de 2017 